ACTA ARITHMETICA

$\mathrm{V}(1959)$

\title{
An arithmetic property of groups of linear transformations
}

by

K. MAHLER (Manchester)

The connection between the geometrical theory of the modular group and that of the minima of binary quadratio forms is classical and well known. In the present note, I study the analogous problem for groups of linear transformations

$z \rightarrow \frac{\alpha_{k} z+\beta_{k}}{\gamma_{k} z+\delta_{k}} \quad\left(\alpha_{k}, \beta_{k}, \gamma_{k}, \delta_{k}\right.$ real $\left.; \alpha_{k} \delta_{k}-\beta_{k} \gamma_{k}=1 ; k=1,2,3, \ldots\right)$

the fundamental region of which is compact in the hyperbotic plane. By means of a theorem due to G. A. Hedlund, it will be proved that if $f(u, v)$ is any positive definite quadratic form, then the values

$$
f\left(\alpha_{k}, \gamma_{k}\right) \quad\left(k_{k}=1,2,3, \ldots\right)
$$

lie dense on the positive real axis. In the cases where the arithmetic structure of the coefficients $\alpha_{k}, \beta_{k}, \gamma_{k}, \delta_{k}$ is known, one is thus led to new arithmetic properties of quadratic forms.

1. Let $\Psi$ be the open complex upper half-plane

$$
y>0, \quad \text { where } \quad z=x+y i,
$$

and let $U$ be its frontier consisting of

(i) the line $y=0$,

(ii) the point $z=\infty$.

We interpret $\Psi$ in the usual way as the non-Euclidean hyperbolic plane, and $U$ as the line at infinity of this geometry.

The hyperbolic lines consist of (i) all semi-circles in $\Psi$ with their centres on $U$, and (ii) all semi-lines in $\Psi$ perpendicular to $U$. These lines are permuted under the rigid motions of hyperbolic geometry which, in explicit form, are defined by the linear transformations

$$
S: z \rightarrow \frac{\alpha z+\beta}{\gamma z+\delta}
$$


of $\Psi$ into itself; here the coefficients $\alpha, \beta, \gamma, \delta$ are real numbers which may be assumed of determinant

$$
a \delta-\beta \gamma=1 .
$$

The rigid motions leave the hyperbotic distance $d\left(z_{1}, z_{2}\right)$ of any two points $z_{1}=x_{1}+y_{1} i$ and $z_{2}=x_{2}+y_{2} i$ in $\Psi$ invariant. If suitably normed, this distance is given by the equation

$$
\sinh \frac{d\left(z_{1}, z_{2}\right)}{2}=\sqrt{\frac{\left(z_{1}-x_{2}\right)^{2}+\left(y_{1}-y_{2}\right)^{2}}{4 \cdot y_{1} y_{2}}} .
$$

In hyperbolic geometry there are three distinct types of oiroles, dopending on their numbers of points of intersection with the line $U$. We are mainly interested in the horocycles which are those hyperbolic circles that meet $U$ in just one point. There are two distinct types of horocycles, viz. (i) those of the form

$$
(x-a)^{2}+(y-b)^{2}=b^{2}, \quad \text { where } \quad b>0,
$$

which are tangent to $U$ at a finite point $z=a$, and $(i i)$ those of the form

$$
y=b, \quad \text { where } \quad b>0,
$$

which may be considered as being tangent to $U$ at $z=\infty$. The rigid motions of the hyperbolic plane change horocycles again into horocycles. In particular, the horocycles of the second form are invariant under the special motions

$$
W: z \rightarrow z+\beta \quad(\beta \text { real }) \text {. }
$$

2. Let $F$ be any Fuchsian group of linear transformations $S$ fo $\Psi$ into itself. A horocycle $C$ is said to be transitive with respect to $F$ if its imagos $S(C)$ under all the elements $S$ of $F$ lie everywhere dense in $\Psi$. In explicit form, if $z_{0}$ is any point in $\Psi$, and

$$
V: d\left(z, z_{0}\right)<\varepsilon, \quad \text { where } \varepsilon>0,
$$

is an arbitrary neighbourhood of $z_{0}$, then at least one imago $\mathbb{S}(\theta)$ of $O$ with $S \in F$ passes through this neighbourhood.

The theory of transitive horocycles has been studied in detail by G. A. Hedlund [1], and one of his results forms the bassis for this paper. Let us, for shortness, say that the Fuchsian group $t^{\prime}$ is admissible if it possesses a compact fundamental region; i. e. IT has a fundamental region, $R_{0}$ say, which lies entirely in $\Psi$ and does not meet the line $U$.

By way of example, every Schwarzian triangle group in $\Psi$ which is generated by a triangle with positive angles is admissible. The modular group, however, is not admissible, because its fundamental regions either contain the point $z=\infty$ or an equivalent point on $U$.

Theorem 2.5 of Hedlund's paper implies the following important result.

THEOREM 1. If the Fuchsian group $F$ is admissible, then every horocycle $C$ in $\Psi$ is transitive with respect to $F$.

In the example of the modular group this theorem does, of course, not hold, and it is, in fact, obvious that no horocycle $y=b$ can be transitive.

3. We need a result which is slightly stronger than Theorem 1 for the application to quadratic forms.

THEOREM 2. Let $F$ be an admissible Fuchsian group, and let $b_{1}$ and $b_{2}$ be real numbers such that $0<b_{1}<b_{2}$. For every point $z_{0}$ in $\Psi$ there exists an element $S$ of $F$ such that $S\left(z_{0}\right)=z_{1}=x_{1}+y_{1} i$ satisfies the inequality

$$
b_{1}<y_{1}<b_{2} \text {. }
$$

Proof. Choose an arbitrary real number $b^{*}$ such that $b_{1}<b^{*}<b_{2}$. There is then a positive constant $\varepsilon$ such that every circular disc

$$
d\left(z, z^{*}\right)<\varepsilon
$$

of radius $\varepsilon$ and with its centre $z^{*}$ on the horocycle $y=b^{*}$ lies entirely in the horizontal strip

$$
b_{1}<y<b_{2}
$$

between the two horocycles $y=b_{1}$ and $y=b_{2}$. By Theorem 1 , the horocycle $C^{*}: y=b^{*}$ is transitive. There is then a transformation $S_{1} \in F$ such that the image $C^{* *}=S_{1}\left(C^{*}\right)$ of $C^{*}$ passes through the disc

$$
d\left(z, z_{0}\right)<\varepsilon .
$$

Choose an arbitrary $z^{* *}$ in $C^{* *}$ satisfying

and put

$$
d\left(z^{* *}, z_{0}\right)<\varepsilon
$$

$$
S=S_{1}^{-1}, \quad z^{*}=S z^{* *}, \quad z_{0}^{*}=S z_{0},
$$

so that also $S \in F$. By the invariance and the symmetry of the hyperbolic distance, also

$$
d\left(z^{*}, z_{0}^{*}\right)=d\left(z_{0}^{*}, z^{*}\right)<\varepsilon
$$

Since $z^{* *}$ lies on $C^{* *}, z^{*}$ lies on the original horocycle $C^{*}$. It follows that $S\left(z_{0}\right)$ is situated in some disc of radius $\varepsilon$ with its centre $z^{*}$ on $y=b^{*}$; hence this point $S\left(z_{0}\right)$ satisfies the assertion. 
4. As before, let $F$ be an admissible Fuchsian group, and let

$$
S_{k}: z \rightarrow \frac{\alpha_{k} z+\beta_{k}}{\gamma_{k} z+\delta_{k}} \quad(k=1,2,3, \ldots)
$$

be all its elements; here $\alpha_{k}, \beta_{k}, \gamma_{k}, \delta_{k}$ are real numbers such that

$$
\alpha_{k} \delta_{l k}-\beta_{k} \gamma_{k}=1
$$

We associate with $S_{k}$ the homogeneous linear transformation

$$
S_{k}^{*}: u \rightarrow \alpha_{k} u+\beta_{k} v, \quad v \rightarrow \gamma_{k} u+\delta_{k} v
$$

Next let

$$
f(u, v)=a u^{2}+2 b u v+c v^{2}
$$

be any binary positive definite quadratic form; hence

$$
a>0, \quad c>0, \quad d=a c-b^{2}>0 .
$$

The transformation $S_{k}^{*}$ changes $f(u, v)$ into a new positive definite form

$$
f_{k}(u, v)=f\left(\alpha_{k} u+\beta_{k} v, \gamma_{k} u+\delta_{k} v\right)=a_{k} u^{2}+2 b_{k} u v+c_{k} v^{2}
$$

with coefficients

$$
a_{k}=f\left(\alpha_{k}, \gamma_{k}\right), \quad b_{k}=a \alpha_{k} \beta_{k}+b\left(\alpha_{k} \delta_{k}+\beta_{k} \gamma_{k}\right)+c \gamma_{k} \delta_{k}, \quad c_{k}=f\left(\beta_{k}, \delta_{k}\right) .
$$

Again

$$
a_{k}>0, \quad c_{k}>0, \quad a_{k} c_{k}-b_{k}^{2}=a c-b^{2}>0,
$$

with the third relation following from the identity

$$
a_{k} c_{k}-b_{k}^{2}=\left(a c-b^{2}\right)\left(\alpha_{k} \delta_{k}-\beta_{k} \gamma_{k}\right)^{2} .
$$

Put

$$
\omega=\frac{-b+i \sqrt{a c-b^{2}}}{a}, \quad \omega_{k}=\frac{-b_{k}+i \sqrt{a c-b^{2}}}{a_{l c}},
$$

where the square root is taken with the positive sign; thus both $\omega$ and $\omega_{k}$ lie in $\Psi$. These two numbers may also be defined as the roots in. $\Psi$ of the two quadratic equations

$$
f(z, 1)=0 \quad \text { and } \quad f_{k}(z, 1)=0,
$$

respectively. Therefore

$$
0=f_{k}\left(\omega_{k}, 1\right)=f\left(\alpha_{k} \omega_{k}+\beta_{k}, \gamma_{k} \omega_{k}+\delta_{k}\right)=\left(\gamma_{k} \omega_{k}+\delta_{k}\right)^{2} f\left(\frac{\alpha_{k} \omega_{k}+\beta_{k}}{\gamma_{k} \omega_{k}+\delta_{k}}, 1\right)
$$

where evidently

$$
\gamma_{k} \omega_{k}+\delta_{k} \neq 0, \text { hence } f\left(\frac{\alpha_{k} \omega_{k}+\beta_{k}}{\gamma_{k} \omega_{k}+\delta_{k}}, 1\right)=0 .
$$

Thus

$$
\omega=\frac{a_{k} \omega_{k}+\beta_{k}}{\gamma_{k} \omega_{k}+\delta_{k}}=S_{k}\left(\omega_{k}\right)
$$

and conversely,

$$
\omega_{k}=\frac{\delta_{k} \omega-\beta_{k}}{-\gamma_{k} \omega+\alpha_{k}}=S_{k}^{-1}(\omega)
$$

$S_{k}^{-1}$ denotes the inverse of $S_{k}$. As $S_{k}$ runs over all elements of $F$, its inverse $S_{k}^{-1}$ does the same since $F$ is a group.

5. Write

$$
\omega=\xi+\eta i \quad \text { and } \quad \omega_{k}=\xi_{k}+\eta_{k} i
$$

so that

$$
\xi=-\frac{b}{a}, \quad \eta=\frac{\sqrt{a c-b^{2}}}{a} \quad \text { and } \quad \xi_{k}=-\frac{b_{k}}{a_{k}}, \quad \eta_{k}=\frac{\sqrt{a c-b^{2}}}{a_{k}} .
$$

On applying Theorem 2, we obtain the following result. If again

$$
0<b_{1}<b_{2}
$$

then there exists a transformation $S=S_{k}^{-1}$ in $F$ such that the imaginary part $\eta_{k}$ of $\omega_{k}=S_{k}^{-1}(\omega)$ satisfies the inequality

$$
b_{1}<\dot{\eta}_{k}<b_{2} \text {. }
$$

This means that

$$
a_{k}=f\left(\alpha_{k}, \gamma_{k}\right)
$$

satisfies the inequality

$$
\frac{\sqrt{a c-b^{2}}}{b_{2}}<a_{k}<\frac{\sqrt{a c-b^{2}}}{b_{1}} .
$$

Since the constants $b_{1}$ and $b_{2}$ are still at our disposal, the result so proved may be expressed as follows.

THEOREM 3. Let $F$ be an admissible Fuchsian group consisting of the linear transformations

$$
s_{k}: z \rightarrow \frac{\alpha_{k} z+\beta_{k}}{\gamma_{k} z+\delta_{k}} \quad(k=1,2,3, \ldots)
$$


where $a_{k}, \beta_{k}, \gamma_{k}, \delta_{k}$ are real numbers satisfying

$$
\alpha_{k} \delta_{k}-\beta_{k} \gamma_{k}=1 \text {. }
$$

Let further

$$
f(u, v)=a u^{2}+2 b u v+c v^{2}
$$

be an arbitrary positive definite quadratic form. The values

$$
f\left(\alpha_{k}, \gamma_{k}\right) \quad(k=1,2,3, \ldots)
$$

of this form lie everywhere dense in the positive real axis.

This theorem may, of course, not be applied to the modular group for which, in fact, the values $f\left(a_{k}, \gamma_{k}\right)$ have no finite limit point.

I conjecture that if the form $f(u, v)$ is indefinite and $T$ is again admissible, then the values $f\left(a_{k}, \gamma_{k}\right)$ lie everywhere dense on both the positive and the negative real axis.

6. Theorem may be applied to many known Fuchsian groups. As an example we consider a group which is related to the theory of indefinite ternary quadratic forms (see Fricke-Klein [2], p. $533 \mathrm{ff}$ ).

Let $p, q, r$ be three squarefree positive integers which are relatively prime in pairs and are such that the ternary form

$$
f\left(z_{1}, z_{2}, z_{3}\right)=p z_{1}^{2}-q z_{2}^{2}-r z_{3}^{2}
$$

is distinct from zero for all integers $z_{1}, z_{2}, z_{3}$ except $z_{1}=z_{2}=z_{3}=0$. We denote $(a, b, c, d)$ all integral solutions of the quaternary Pellian equation

$$
a^{2}-b^{2} p r+c^{2} q r-d^{2} p q=4 .
$$

It is then proved that the unimodular transformations

$$
s: z \rightarrow \frac{\alpha z-\beta}{\gamma z-\delta} \quad(\alpha \delta-\beta \gamma=1)
$$

where

$$
\begin{array}{ll}
a=\frac{a+b \sqrt{p r}}{2}, & \beta=\frac{a \sqrt{r}+a \sqrt{p}}{2} \sqrt{q}, \\
\gamma=\frac{-c \sqrt{r}+d \sqrt{p}}{2} \sqrt{q}, & \delta=\frac{a-b \sqrt{p r}}{2}
\end{array}
$$

form an admissible Fuchsian group. We deduce therefore at once from Theorem 3 that if again $f(u, v)$ is any positive definite quadratic form, then the values

$$
f\left(\frac{a+b \sqrt{p r}}{2}, \frac{-c \sqrt{r}+d \sqrt{p}}{2} \sqrt{q}\right)
$$

lie dense on the positive real axis.

It seems probable that Theorem 3 has an analogue for Kleinian polyhedron groups and positive definite Hermitean form.

\section{References}

[1] G. A. Hedlund, Fuchsian groups and transitive horocycles, Duke Math. J. 2 (1936), p. $530-542$. 1897.

[2] R. Fricke und F. Klein, Automorphe Funktionen, Band 1, Leipzig

MATHEMATICS DEPARTMENT, MANCHESTER UNIVERSITY 28 August, 1958

Regu par la Rédaction le 12.9. 1958 